\title{
National shortage of tuberculin skin test antigens
}

\author{
David Sotello MD
}

Adequate identification and treatment of patients with latent tuberculous infection (LTI) is a matter of high importance in public health. Persons with the highest risk factor(s) for LTI include close contacts with active tuberculosis cases, drug users, immigrants from certain areas, health care workers, immunocompromised patients, and patients with renal failure, etc.

The two most common methods of identifying LTI cases are the tuberculin skin test (TST) and the interferon-gamma release assay (IGRA). It is important to remember that neither tests can differentiate between active or latent tuberculous infection.

The TST is an intradermal injection of tuberculin material that stimulates a T cell response in those previously infected with Mycobacterium tuberculosis. The TST has been used for multiple years and has a low cost. The main limitations with its use is the requirement for a follow up visit to evaluate the result at $48-72$ hours, misinterpretation secondary to reader error, the presence of anergy, cross-reaction with other antigens (e.g., non-tuberculous mycobacteria), and a false positive result following Bacillus CalmetteGuerin vaccination.

The IGRAs are in vitro blood tests that measure $\mathrm{T}$ cell responses to $M$ tuberculosis antigens,

Corresponding author: David Sotello Contact Information: David.sotello@ttuhsc.edu DOI: $10.12746 /$ swrccc.v7i30.570 have higher sensitivity and specificity than TST, do not require a follow-up visit, but have a higher cost. Some results can be classified as indeterminate or borderline depending of the assay used and the host immune status, and this creates uncertainty.

In June 2019, the CDC issued a communication that stated that there is currently a nationwide shortage of Aplisol $^{\circledR}$, one of two purified-protein derivate TST antigens; this shortage could last 3-10 months. The CDC recommends substituting IGRAs for TSTs. Another option is to substitute Tubersol ${ }^{\circledR}$ for Aplisol ${ }^{\circledR}$ for skin testing, if available. A third option is to prioritize allocation of TSTs for those with higher risk for $\mathrm{LTI}$. It is important to consider these recommendations when evaluating patients at risk for LTBI.

Keywords: tuberculosis, skin tests, interferon gamma release assays

Submitted: $7 / 4 / 2019$

\section{REFERENCE}

1. Nationwide shortage of tuberculin skin test antigens: CDC recommendations for patient care and public health practice. MMWR Morb Mortal Wkly Rep 2019;68:552-553. 